\title{
Design of an under-actuated wrist based on adaptive synergies
}

\author{
Simona Casini ${ }^{2}$, Vinicio Tincani ${ }^{2}$, Giuseppe Averta ${ }^{1}$, Mattia Poggiani ${ }^{1}$, Cosimo Della Santina ${ }^{1}$, \\ Edoardo Battaglia ${ }^{1}$, Manuel G. Catalano ${ }^{2}$, Matteo Bianchi ${ }^{1}$, Giorgio Grioli ${ }^{2}$ and Antonio Bicchi ${ }^{1,2}$
}

\begin{abstract}
An effective robotic wrist represents a key enabling element in robotic manipulation, especially in prosthetics. In this paper, we propose an under-actuated wrist system, which is also adaptable and allows to implement different underactuation schemes. Our approach leverages upon the idea of soft synergies - in particular the design method of adaptive synergies - as it derives from the field of robot hand design. First we introduce the design principle and its implementation and function in a configurable test bench prototype, which can be used to demonstrate the feasibility of our idea. Furthermore, we report on results from preliminary experiments with humans, aiming to identify the most probable wrist pose during the pre-grasp phase in activities of daily living. Based on these outcomes, we calibrate our wrist prototype accordingly and demonstrate its effectiveness to accomplish grasping and manipulation tasks.
\end{abstract}

\section{INTRODUCTION}

In humans, the wrist is responsible for fine adjustments of hand pose [1], [2], [3] and plays a fundamental role in hand mobility and manipulation in everyday actions [4]. The development of effective robotic wrists is also very important in robotic manipulation, since the wrist enables the control of the end-effector pose. This is crucial in many applications, ranging from assistive robotics and prosthetics, human-robot interaction, micro-manipulation for medical procedures, orienting machine tools, see e.g. [5], [6], [4]. Furthermore, within the novel paradigm of soft manipulation, i.e. robot embodied ability to comply and adapt to features of the environment, an effective design of robotic wrists is an essential component to simplify the grasp planning phase. Indeed, leveraging upon the intrinsic capabilities of soft end effectors to continuously deform in different shapes, an approximation of object properties and hand pose definition, which is ultimately determined by the wrist, can be enough to generate candidate poses to grasp an external item [7]. Among those application fields, prosthetics probably represents one of the most challenging, due to the significant contribution that a robotic wrist can provide in terms of users quality of life and prosthesis acceptance [4] and due to the additional constraints that must be taken into account during the design phase. Interestingly, while important advancements for the development of dexterous

*This work was supported by the European Commission projects (Horizon 2020 research program) SOFTPRO (no. 688857) and SOMA (no. 645599) and by the European Research Council under the Advanced Grant SoftHands "A Theory of Soft Synergies for a New Generation of Artificial Hands" (no. ERC-291166)

${ }^{1}$ Centro di Ricerca "E. Piaggio", Univ. di Pisa, Italy.

${ }^{2}$ Dept. of Adv. Robotics, Istituto Italiano di Tecnologia, Genoa, Italy

Correspond to: simona.casini at iit.it

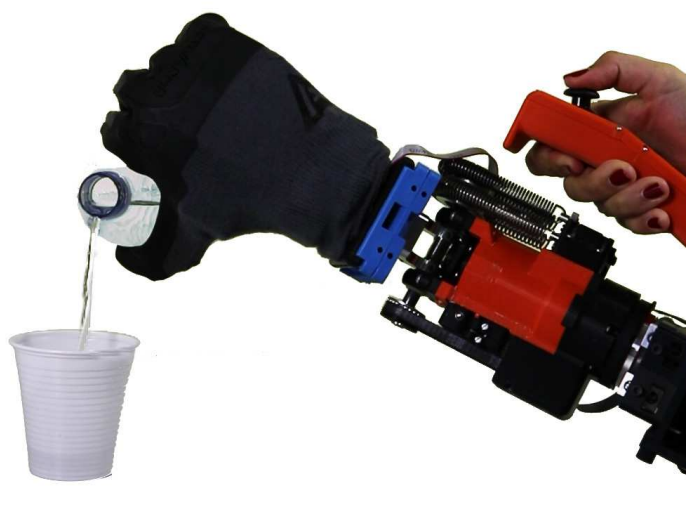

Fig. 1. The two DOFs adaptive under-actuated wrist pouring water.

hands for prosthetic use have been done, wrist design has drawn much less interest among researchers and modest progresses can be observed so far. Indeed, only few examples of commercially available prosthetic wrists can be found, including both motorized solutions for hand closure and pronation/supination (P-S), flexion/extension (F-E) and finger closing, as well as passive solutions (see [8] for a review on these topics). Considering also research developments, we can find several architectures, including passive wrists (see e.g. [9]), 2 DOFs fully-motorized wrists (see e.g. [10], [11]), 3 DOFs fully-motorized wrist (see e.g. [12]), rotators [13], body-powered wrists [14], among the others (refer to [4] for a complete review).

In this work, we present the mechanical design of a soft and adaptable under-actuated wrist prototype with 2 DOFs (see Fig.1). Thanks to its modularity, this system allows to implement different under-actuation patterns and represents a useful test-bed to shape the development of other prototypes with reduced encumbrance. Since the absence of P-S is very penalizing to be compensated for (because it requests a rotation of the shoulder around the axis of the arm), we decided to implement this DOF, while our architecture allows to choose between F-E and add-abduction (A-A), or a combination of the two, as the second DOF. At the same time, we also developed procedure to retrieve human upper limb kinematics from optical marker-based measurements and performed preliminary experiments with human participants to identify upper limb joint angles and extract the most probable wrist pose at the moment of the pre-grasp.. This pattern was then implemented in our robotic 
wrist. Experiments where the wrist was used in conjunction with the Pisa/IIT SoftHand [15] to grasp different items show the effectiveness of our solution, in terms of grasp success, opening scenarios for robotic, prosthetic and rehabilitative applications [16].

\section{A WRIST WITH SYNERGIES}

The theoretical basis for the proposed modular underactuation moves from the concept of adaptive synergies [15], which combines synergy-based actuation and mechanical adaptability for the development of soft robotic hands. This approach can be applied also to the simplification of the design of modular wrists. Furthermore, the proposed modular approach enables the implementation and testing of different under-actuation strategies, which in turn can take inspiration from human example.

In neuroscience the concept of synergies, i.e. multiple elements working together towards a common goal, has been extensively studied and developed as a principled simplification approach to the well-known Bernstein's problem of motor redundancy [17]. Although synergies can be defined at different levels, e.g. kinematic, muscular, neural and also sensory (see e.g. [18], [19] ), the main idea shared across these definitions is the geometric concept of dimensionality reduction, i.e. multiple degrees of freedom can be controlled within a lower dimensional space than the available number of dimensions. Such a descriptive framework has been applied to investigate how the central nervous system can control the large number of sensory-motor elements of the hand, such as ligaments, muscles, receptors, joints and bones. At the same time, the existence of a co-variation (i.e. a synergy) for the joint angles of the whole upper limb has been also experimentally proven in literature. For example, previous works demonstrated that movements of the arm and shaping of the hand during reach-to-grasp are highly coordinated [20], and hence that a global control strategy for the coordination of the fingers, wrist and arm can be observed [21], [22]. Focusing on grasping and manipulation, the control of hand position and orientation w.r.t the external object represents a key factor for enabling a correct task accomplishment and can be regarded as the result of coordination and contribution of different upper-limb parts, such as the shoulder, the elbow, and finally, the wrist.

At the same time, robotics research has deeply leveraged upon these neuroscientific insights for the design of artificial hands and sensing systems with a reduced number of actuators, control inputs, and sensors (see e.g. [15], [23], [24], [19]).

More specifically, soft synergies [25], [26] were proposed as a tool for modeling human motor behavior and driving the design of artificial hands. According to this framework, a reference configuration can be generated using a reduced sub-set of all the possible degrees of freedom (called postural synergies [19]), while the real limb is compliantly attracted to it and, at the same time, repelled by the forces of interaction with the external world. When an equilibrium is reached, we

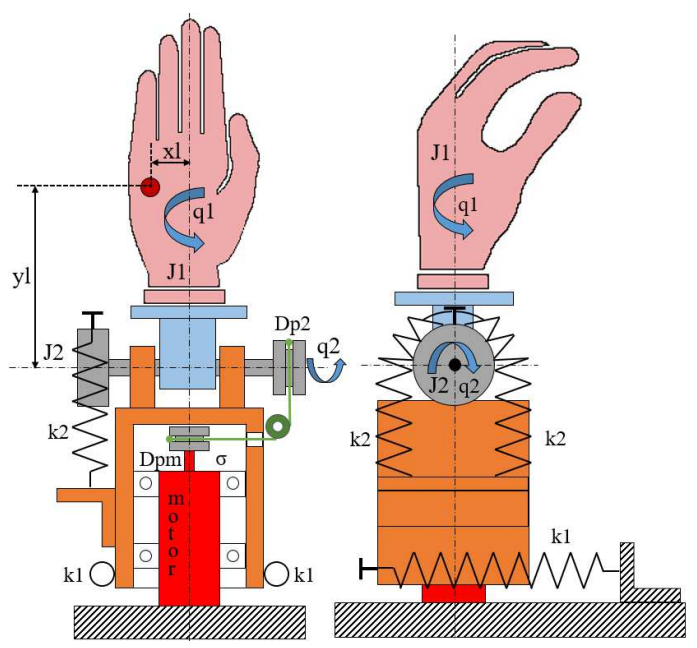

Fig. 2. Wrist model used for the mechanical design.

have

$$
q=S \sigma-C J^{T} f_{\mathrm{ext}},
$$

where $\sigma \in \mathbb{R}^{s}$ are the synergy variables, $q \in \mathbb{R}^{n}$ are the joint variables. $S \in \mathbb{R}^{n \times s}$ represents the synergistic basis of the joints configuration space, mapping $\sigma$ into $q$. The matrix $C \in \mathbb{R}^{n \times n}$ is the compliance and $J^{T} f_{\text {ext }} \in \mathbb{R}^{n}$ collects the effect of all the external forces acting on the system.

Starting from these considerations, adaptive synergies [27], [15] were proposed as a design paradigm to implement soft synergies leveraging upon the adaptability of differential mechanism. In this case, let $R \in \mathbb{R}^{s \times n}$ be the transmission distribution matrix and $E \in \mathbb{R}^{n \times n}$ the joint space stiffness matrix. The equilibrium of the joint torques, which includes a linear elastic force in joint space $-E q \in \mathbb{R}^{n}$ and the contribution of external forces $J^{T} f_{\text {ext }} \in \mathbb{R}^{n}$, is

$$
\left\{\begin{array}{l}
J^{T} f_{\mathrm{ext}}=R^{T} f-E q \\
R q=\sigma .
\end{array}\right.
$$

where $f \in \mathbb{R}^{s}$ is the generalized force produced by the motor. Solving Eq. (2) leads to Eq. (1) with the following choice of matrices (see [27] for more details)

$$
\begin{aligned}
& S=E^{-1} R^{T}\left(R E^{-1} R^{T}\right)^{-1} \\
& C=E^{-1}-E^{-1} R^{T}\left(R E^{-1} R^{T}\right)^{-1} R E^{-1} .
\end{aligned}
$$

Through this relation the matrices $S$ and $C$ are related to the mechanical elements represented by $E$ and $R$. Thus both un-perturbed movements and elastic behavior of the robot can be independently designed by proper choice of elastic constant of the springs and the radii of the pulleys. This framework was successfully applied in the design of robotic hands [15], [28], [29]. Naturally conforming to the external environments in a human-like manner, such hands are able to perform complex grasps and basic in-hand manipulations with a small set of control inputs and an intuitive interface.

As described above such characteristics are highly attractive for a robotic wrist. In the authors' opinion, passive adaptability of the wrist should help the user providing 
an intuitive way to take advantages from environment, in order to achieve more stable hand pre-orientations, and to perform manipulation tasks. For these reasons, in this work we propose an adaptive synergy-based robotic wrist, which allows us to investigate these ideas, keeping intentionally separated the wrist synergies from other possible synergies implemented in the artificial hand mounted on it.

Furthermore, the modularity of our prototype enables the implementation and testing of different under-actuation patterns. In our first prototype we consider just the two DOFs $q_{1}, q_{2}$ rotating around axes $J_{1}, J_{2}$, instead of the three DOFs which are usually considered to model the human wrist. The two DOFs endowed in the wrist are P-S and F-E in the configuration shown in Fig.2. Main motivation for this relies on results from preliminary human studies, described more in detail in section IV, which revealed that a suitable combination of these two DOFs is enough to explain around $95 \%$ of wrist pose variability during the pre-grasp phase in daily life activities. In addition, a DOF reduction generally implies space and weight decrease which are well liked especially for applications in prosthetics. However, it is worth to note that the orientation of the hand w.r.t. the wrist can be changed, which causes $J_{2}$ to switch between F-E and A-A, as better explained in Sec. III. Fig.2 shows the main components of the proposed system.

The elastic field $E q$ is here implemented through the springs of elastic constant $k_{1}$ and $k_{2}$, while the transmission ratio $R$ is implemented by the pulleys of pitch diameter $D_{p 2}$ and $D_{p m}$. The next section provides a more accurate description of the actual mechanics implementing the described components.

We call $x_{G}$ and $x_{L}$ respectively the distance between $J 1$ and the center of mass $G$ of the hand and an applied load $L$ normal to the palm of the hand and intensity $f_{\mathrm{L}}$ and similarly $y_{G}$ and $y_{L}$ the distance between $J 2$ and $G$ and $L$. Then Eq. (2) for the proposed wrist is

$$
\left[\begin{array}{ll}
x_{G} & x_{L} \\
y_{G} & y_{L}
\end{array}\right]\left[\begin{array}{c}
M_{h} g \\
f_{\mathrm{L}}
\end{array}\right]=\left[\begin{array}{c}
1 \\
D_{p 2} \\
\frac{D_{p m}}{2}
\end{array}\right] f-\left[\begin{array}{cc}
k_{1} & 0 \\
0 & k_{2}
\end{array}\right]\left[\begin{array}{l}
q_{1} \\
q_{2}
\end{array}\right],
$$

being $M_{h}$ the mass of the hand. Thus (3) leads to the following description of wrist behavior

$$
\begin{aligned}
q= & {\left[\begin{array}{c}
D_{p m} k_{2} \\
D_{p 2} k_{1}
\end{array}\right] \frac{\sigma D_{p m}}{k_{1} D_{p 2}^{2}+k_{2} D_{p m}^{2}}+} \\
& {\left[\begin{array}{ll}
\frac{D_{p 2}^{2}}{k_{1} D_{p 2}^{2}+k_{2} D_{p m}^{2}} & \frac{-D_{p 2} D_{p m}}{k_{1} D_{p 2}^{2}+k_{2} D_{p m}^{2}} \\
\frac{-D_{p 2} D_{p m}}{k_{1} D_{p 2}^{2}+k_{2} D_{p m}^{2}} & \frac{D_{p m}^{2}}{k_{1} D_{p 2}^{2}+k_{2} D_{p m}^{2}}
\end{array}\right]\left[\begin{array}{ll}
x_{G} & x_{L} \\
y_{G} & y_{L}
\end{array}\right]\left[\begin{array}{c}
M_{h} g \\
f_{\mathrm{L}}
\end{array}\right] . }
\end{aligned}
$$

Among the many ways to link the DOFs of the wrist the proposed device reproduces wrist synergies with a direct proportional dependency between $q_{1}$ and $q_{2}$. From equation (4) it can be noted that, for null external loads, the ratio of joint variables is given by the equation $\frac{q_{1}}{q_{2}}=\frac{k_{2}}{k_{1}} \frac{D_{p m}}{D_{p 2}}$. Acting on the ratio of the elastic constants of the springs and on the diameters of the driver and driven pulleys, we can tune off-line a coordinated motion of the DOFs of

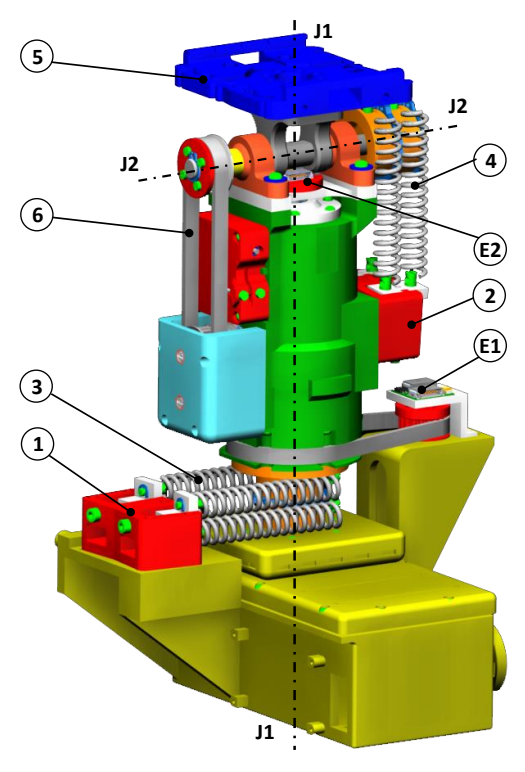

Fig. 3. 3D render of the wrist with the main components of the prototype.

the wrist in absence of external loads (as during the grasp acquisition stage). In the following section, we discuss the mechanical implementation and thoroughly discussed the possible choices for spring constants.

\section{MECHANICAL DESIGN}

Fig.3 shows an overall 3D render of the wrist. The majority of the parts are made in ABS plastic with a FDM machine, whereas the most stressed parts are in Aluminum Alloy or Stainless Steel. The device comprises three main groups:

a) Frame: it is the yellow part located at the bottom of the device. It constitutes the fixed frame on which a joystick can be mounted to handle the wrist (see the pictures of Section V) and contains the electronic components to drive the motors of the hand and the wrist. Group (1), fixed to the frame, enables the loading of springs (3) at axis $J_{1}$. Encoder (E1) directly measures the P-S angle $q_{1}$ with a transmission ratio $2.5: 1$.

b) Pronation-Supination Group: it is the main cylindrical body which confers the P-S movement to the wrist (axis $J_{1}$ ), with the motor embedded. Group (2) enables the loading of springs (4) wrapping around axis $J_{2}$ while toothed belt (6) transmits the power from the motor to $J_{2}$. On the top of the cylindrical body, encoder (E2) measures the relative angle between the motor shaft and the cylindrical body $\sigma-q_{1}$. As shown in Section II, the reading of E2 equals $\frac{q_{2} D_{p 2}}{D_{p m}}$, thanks to which we can get a direct measurement of A-A or FE. A third redundant encoder (E3) housed in the cylindrical body, has the purpose of allowing a direct measurement of the rotation of the motor without being dependent from other encoder readings.

c) Flexion-Extension Group (or Abduction-Adduction Group): it is located at the top of the device and confers a rotation around axis $J_{2}$. At the top of the wrist, flange (5) 


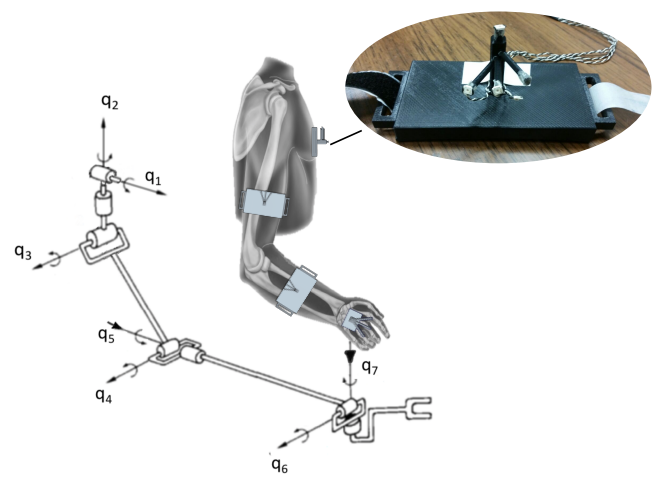

(a) Kinematic Model (adapted from [30]) and marker supports (in light gray and in the zoomed view) used for the experiments.

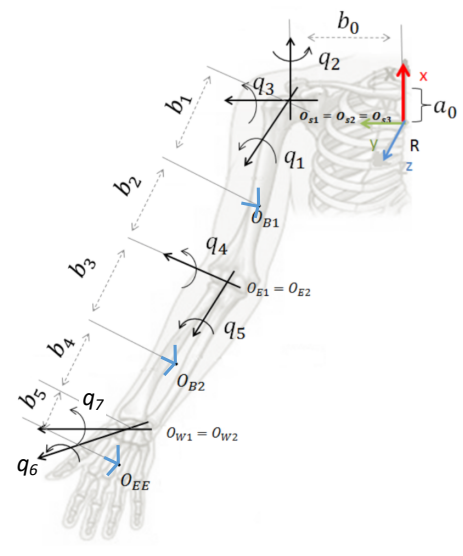

(b) Geometric Parameters.

Fig. 4. Upper limb model used in the identification. Joint variables model shoulder abduction-adduction $\left(q_{1}\right)$, shoulder external-internal rotation $\left(q_{2}\right)$, shoulder flexion-extension $\left(q_{3}\right)$, elbow flexion-extension $\left(q_{4}\right)$, elbow pronosupination $\left(q_{5}\right)$, wrist abduction-adduction $\left(q_{6}\right)$ and wrist flexion-extension $\left(q_{7}\right)$, respectively.

allows to mount the hand with different orientations at $90^{\circ}$. If the hand is mounted as in Fig. 2 the rotation around axis $J_{2}$ is F-E. Conversely, if the hand is rotated $90^{\circ}$ on the flange with respect to Fig.2, the rotation is A-A.

\section{WRIST SYNERGIES IN HUMANS}

One of the motivations of having a modular adaptive wrist is to enable a suitable under-actuation taking inspiration from human example. For this reason, we performed a set of preliminary experiments aiming to understand which are the most probable poses of the human wrist during activities of daily living. In order to carry out these experiments, we defined a kinematic model for the whole upper limb, and an estimation strategy to obtain joint angles from a markerbased optical motion capture system (PhaseSpace, working frequency of $480 \mathrm{~Hz}$ ). While the wrist represents the main interest of this work, tracking is extended to the whole upper limb in order to increase the robustness of the optical motion tracking. Inspired by [30], [31], [32], [33], we modeled the upper limb using three rigid segments and seven DOFs: three DOFs for the shoulder (A-A, F-E and external-internal rotation of the humerus w.r.t the scapula), two for the elbow (F-E of the ulna-radius w.r.t the humerus and P-S due to the relative movement of radius and ulna), and two for the wrist (F-E and A-A of hand w.r.t the forearm). The axes for the shoulder, elbow and wrist joints are assumed to be orthogonal and intersecting.

We used rigid supports with attached markers to define a reference frame on the chest, and local frames in the middle of forearm, arm and hand dorsum as in Fig.4(a). The segments model upper arm, forearm and hand, respectively, and are connected through revolute joints. We chose to use the Product of Exponentials (POE) parametrization to model kinematics [34]. Importantly, in this model the elbow pronosupination degree of freedom, that ultimately determines the pose of the hand, is attributed to the elbow, as was done in [30]. It is worth to note that in our robotic prototype this degree of freedom is considered as part of the wrist. Under a kinematic point of view these two approaches are equivalent in terms of hand pose control. However, our mechanical solution is preferable looking at prosthetic applications, e.g. for trans radial amputees, where hand P-S is controlled at wrist level. In Fig.4(b), we show twist axes for each degree of freedom and other descriptive parameters of the model used during the calibration phase.

Referring to [34], we implemented a calibration procedure to estimate model parameters based on a constrained least-squares minimization between marker positions and model-related marker positions. Joint angle were retrieved implementing a recursive estimation procedure based on an iterative Extended Kalman Filter.

\section{A. Experimental Procedure}

10 right-handed subjects $(6 \mathrm{M}, 4 \mathrm{~F}$, their age ranging from 20 to 30) gave their informed consent to take part to the experiment. No one had any physical limitation that could have affected the experimental outcomes. During the experiment, participants wore the previously described marker supports, and were comfortably seated. They were asked to grasp to use a set of objects as in table I and to perform the related activities of daily living tasks extracted from [35]. Each task was repeated three times and the order of actions

TABLE I

DAILY LIVING ACTIVITIES.

\begin{tabular}{l|l}
\hline 1 & Brush/comb hair \\
2 & Stir in a bowl \\
3 & Cut meat with knife and fork \\
4 & Pour from a bottle/can \\
5 & Write the word "LETTER" legibly \\
6 & Use a pair of scissors \\
7 & Use a key in lock \\
8 & Dial a touch tone phone \\
9 & Use a hammer and nail \\
\hline
\end{tabular}

randomized. In particular, for each action, the object was placed on a table $(\approx 80 \mathrm{~cm}$ height $)$ in front of the participant, 
TABLE II

WRIST SYNERGIES EXTRACTED FROM THE EXPERIMENTS.

\begin{tabular}{c|c|c|c|c} 
& P-S & A-A & F-E & variance \\
\hline PC1 & 0.36 & $4 \cdot 10^{-3}$ & 0.93 & $74 \%$ \\
\hline PC2 & 0.93 & 0.013 & -0.36 & $21 \%$ \\
\hline PC3 & -0.013 & $\sim 1$ & $9.3 \cdot 10^{-4}$ & $5 \%$
\end{tabular}

at a distance of $\approx 50 \mathrm{~cm}$, while the starting hand position was palm-down on the table. This list of actions was performed as trade-off between experimental time and human workspace description, with no claim of exhaustiveness. Study of a more complete list of human actions will be carried out in future works. Note that although we are interested in the pose of the wrist in the pre-grasp phase, we asked participants to complete all the action phases to have more natural data for our analysis.

\section{B. Data Analysis}

Once joint angles were estimated, we focused on the degrees of freedom corresponding to the ones we can implement in our robotic wrist, i.e. $q_{5}, q_{6}$ and $q_{7}$ as in Fig.4. To identify the most common and probable pre-grasp wrist pose, we performed Principal Component Analysis on the data. Wrist orientations were collected in a matrix $A \in \mathbb{R}^{90 \times 3}$, where the number of rows comes from the 10 participants and 9 actions, and the number of columns corresponds to the three degrees of freedom considered. The three repetitions for each action in Tab.I were averaged to privilege, in our PC analysis, the diversity between experimenters and actions rather than variations within the same action performed by the same user. The principal components (PCs) were then computed from the eigenvalues and eigenvectors of the matrix of the covariance coefficients between each of the 90 samples.

Results of the PC analysis are reported in table II.

Although preliminary, these results provided us useful indications on how to control the wrist, i.e. to constrain the degrees of freedom, according to the first common actuation pattern PC1 of the wrist poses in human pre-grasping, which takes into account large part of pose variability. What is noticeable is that according to PC1 F-E plays the most important role: this is not surprising given the set of actions we considered. In the future these results will be further investigated, varying both the location of the object in front of the participant and the typologies of the items used for the experiments.

\section{EXPERIMENTS}

In this Section, we describe a hardware and software setup to prove the device effectiveness in P-S and F-E or A-A movements while grasping different objects with the Pisa/IIT SoftHand. The 2-DOF under-actuated wrist has been provided with a 2-axes joystick hardware interface to handle both the hand and the wrist motor. The thumb-joystick controls the device through a velocity-based control loop, i.e. the farther is the joystick position from the rest the faster is the motor rotation. The joystick is at rest when the thumb is centred on both axes. From this position the SoftHand closes when the joystick is moved upward, while the wrist rotates if it is moved laterally.

Two different sets of experiments have been carried out with this setup: a first group aiming to give a qualitative demonstration of the wrist capabilities, and a second showing the implementation of the $P C 1$.

\section{A. System demonstration}

Fig. 5 and 6 show the wrist free movement in two different configurations. In Fig.5 from the neutral position (center), one can have either pronation and extension (left) or supination and flexion (right). On the contrary, Fig. 6 refers to the case with the hand mounted on the wrist toothed flange to have pronation and adduction (left) or supination and abduction (right). It is of relevance also to have a look on the movement of the device while a DOF is blocked to notice how a pure P-S or a F-E / A-A movement can be obtained (see Fig.7 and Fig.8). Fig.9 shows the under-actuated wrist device while it is used with the thumb-joystick interface for a typical daily living activity. The here proposed device is also capable of exploiting the environment and of passively adapting to improve objects approach and grasping. Fig.10 shows an example of this kind of adaptability, while the user presses the under-actuated wrist on a table to block F-E and to convey only P-S movement. The environmental constrain exploitation is a fundamental aspect not only for a natural usage in assistive robotic applications, but also for soft robotic manipulation, in general. In these pictures it is possible to see the wrist in conjunction with the Pisa/IIT SoftHand [15]. What is noticeable is that the integrated system enables advanced manipulation capabilities, see e.g. Fig.9. Furthermore, it is worth to observe that the orientation of the arm of the user maneuvering our device is minimized during task execution. This is extremely important in view of future prosthetic implementations, to reduce the need of compensatory movements in amputees during the execution of activities of daily living.

\section{B. Implementation of PCs.}

In Section IV we showed a set of preliminary experiments aimed at investigating which are the most probable poses of the human wrist in daily living activities, which led to the individuation of a set of principal components where the first accounts for more than $74 \%$ of variance. In the following we show the implementation of the $P C 1$ in our device and its employment in an applicative case.

Referring to equation (4), the problem of implementing PC1 through hardware is reduced to finding $k_{1}$ and $k_{2}$ such that the ratio between $q_{1}$ and $q_{2}$ is coherent with the ratio assigned by the first principal component. We recall that this $\mathrm{PC}$ has a dominance of the flex-extension joint, with P-S coming second and add-abduction being set to an almost null value. This makes it possible to implement PC1 by assembling the hand in the P-S/F-E configuration (Fig.5) and choosing the elastic constants appropriately. The target ratio 

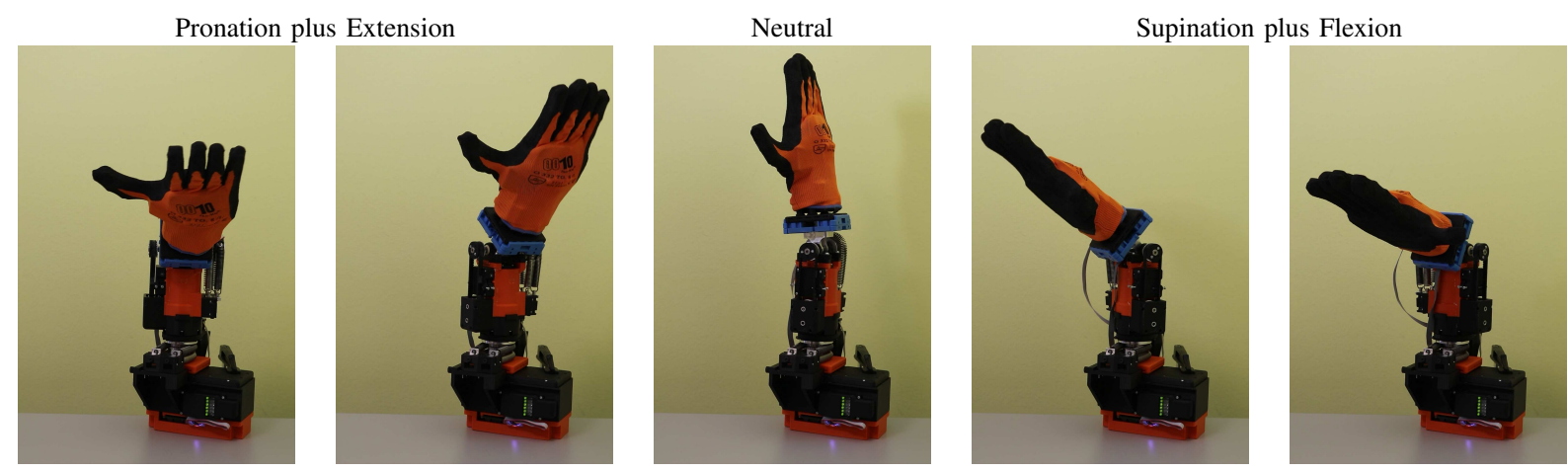

Fig. 5. Free movement of the under-actuated system wrist-SoftHand $\left( \pm 90^{\circ}\right.$ excursion for both P-S and F-E).
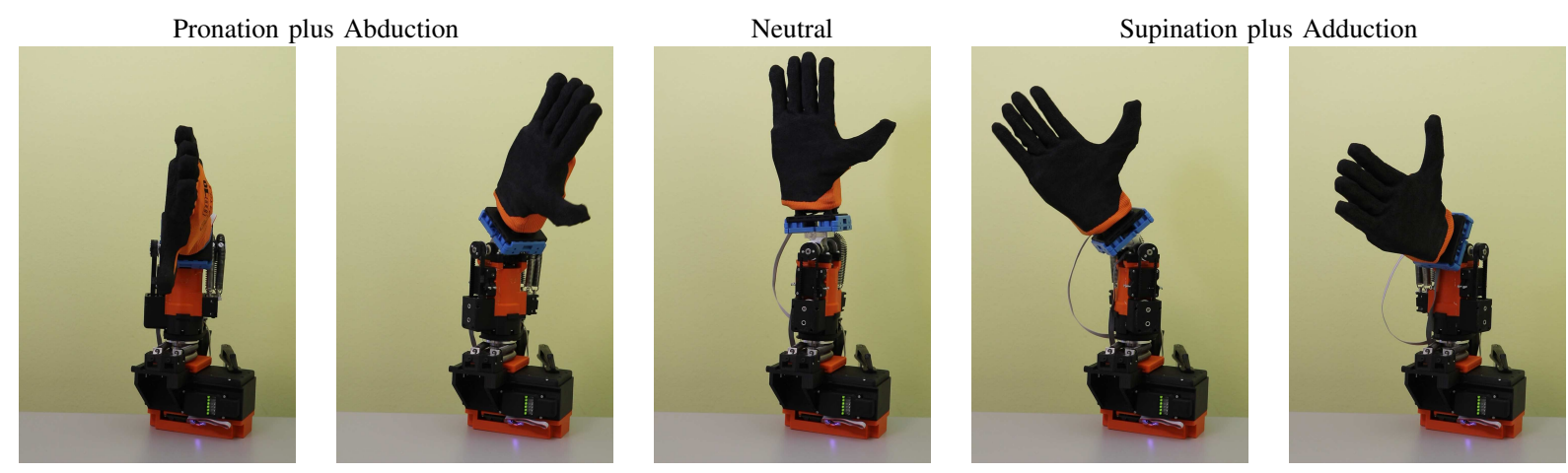

Fig. 6. Free movement of the under-actuated system wrist-SoftHand $\left( \pm 90^{\circ}\right.$ excursion for both P-S and A-A).
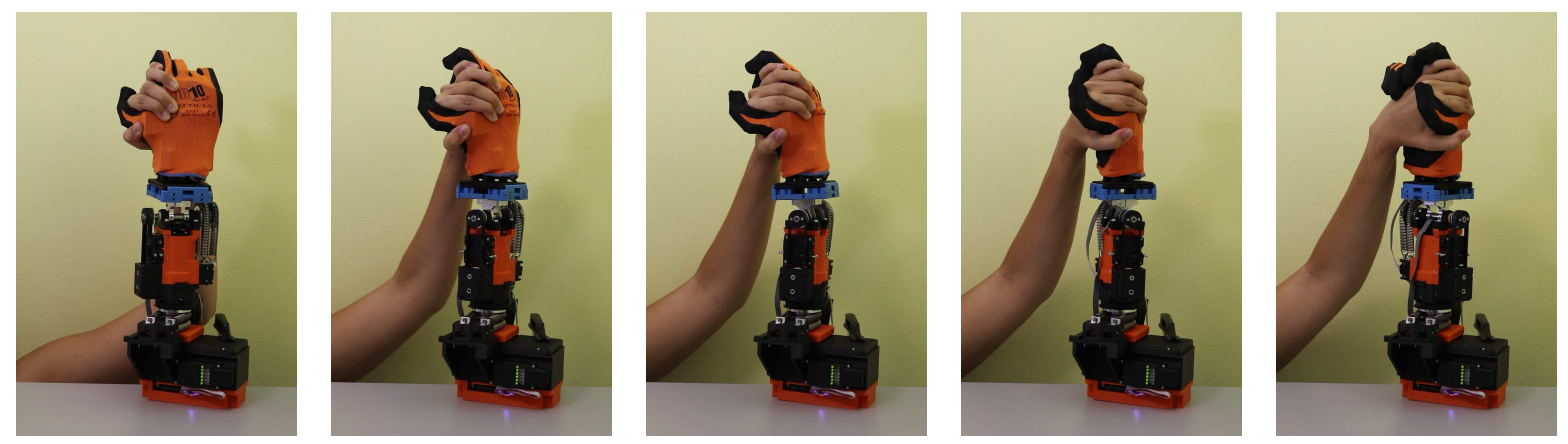

Fig. 7. P-S full range movement while F-E is blocked by an external constraint.
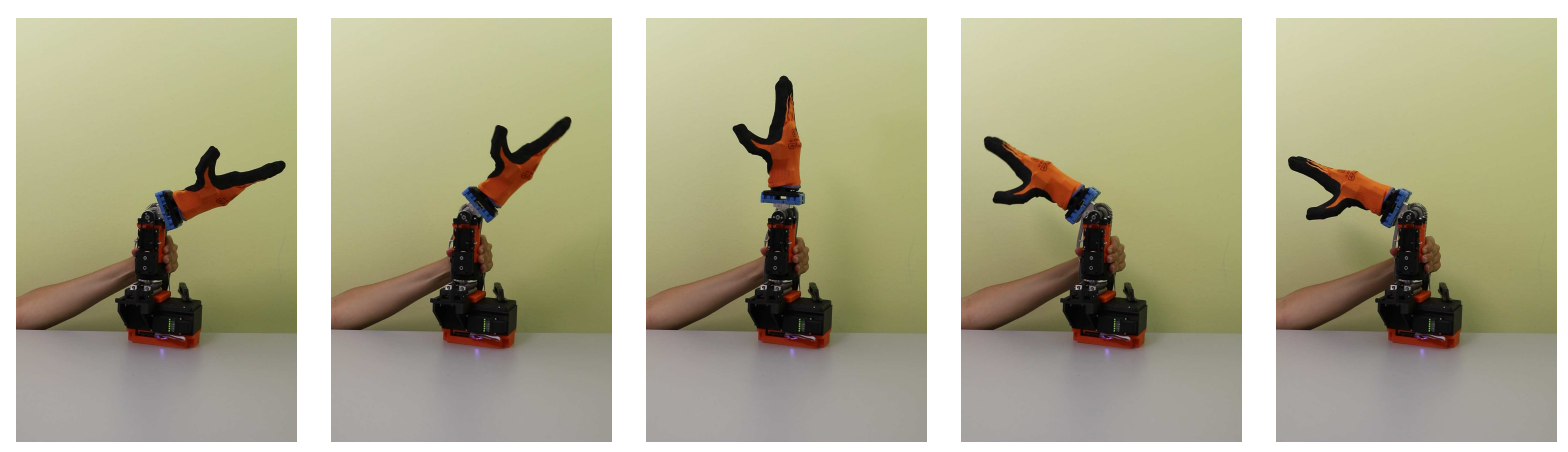

Fig. 8. F-E full range movement while P-S is blocked by an external constraint. 

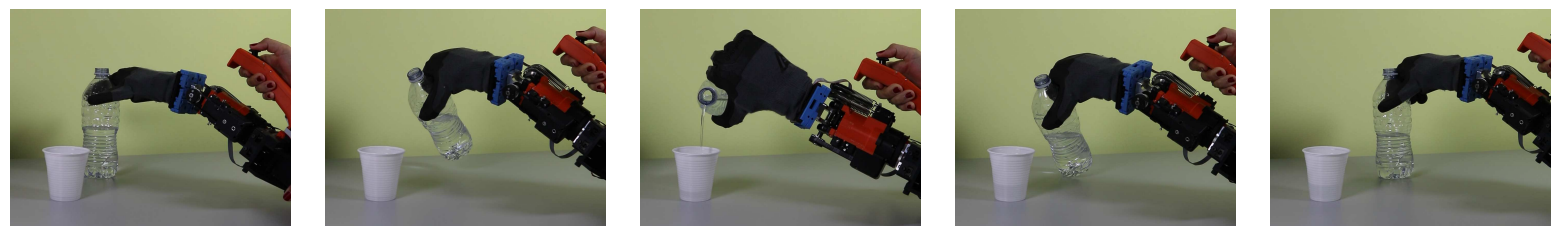

Fig. 9. The wrist driven by a user using thumb-joystick interface to approach and to grasp objects.
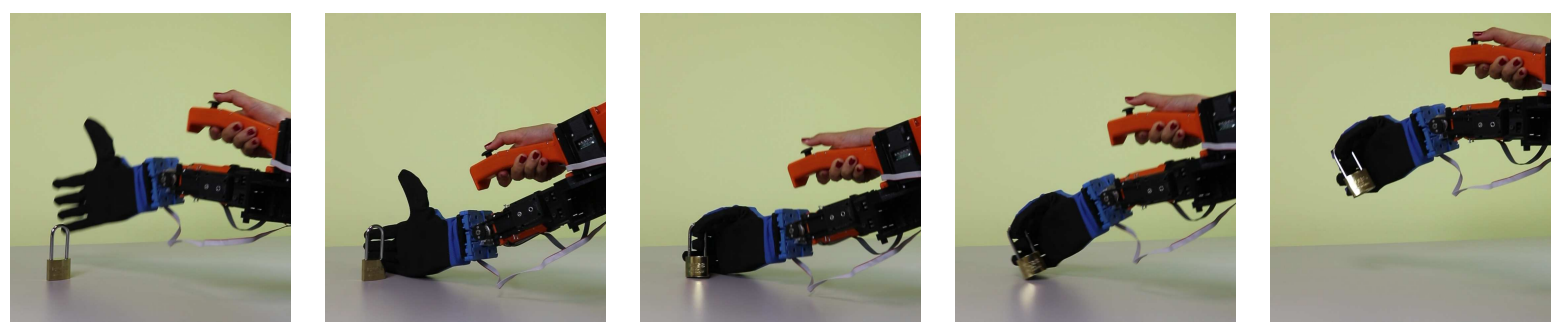

Fig. 10. A typical usage of the here realized device where passive adaptability to environment is effective during task accomplishment.

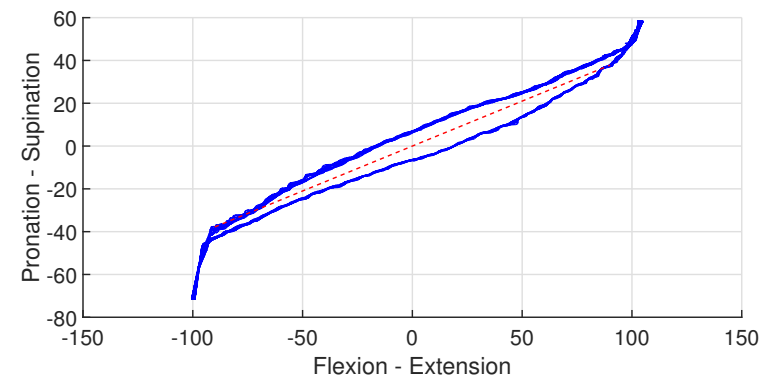

Fig. 11. Joint angles behaviour while PC1 is implemented in the artificial wrist: ideal ratio between F-E and P-S movements (red line) vs. real ratio deduced by 10 cycles experiments (blue lines).

of the P-S and F-E components of the first principal direction (see Tab. II) $\frac{q_{1}}{q_{2}}=\frac{0.36}{0.93} \approx 0.39$ can be implemented in our device with a proper choice of the elastic constant $k 1$ and $k 2$ of the springs. With our available springs at the moment of the experiments we implemented a ratio $\frac{q_{1}}{q_{2}}=0.42$ as the closest approximation $\left(k_{1}=1.8 \mathrm{Nmm}\right.$ and $\left.k_{2}^{q_{2}}=0.55 \mathrm{Nmm}\right)$. The plot of Fig.11 illustrates the $q 1 / q 1$ ratio of the encoder readings after 10 cycles (blue lines). In the same picture the target ratio is overlapped in red dot line showing a good match with the recorded data. The hysteretic behaviour visible in Fig.11 is just due to clearances and static friction in the physical implementation of the wrist.

Fig.12 shows a grasp performed with this PC1 implemented, with all the previously discussed characteristics (i.e. adaptability, enhanced grasping capabilities and absence of compensatory movements) well depicted. Notice that different spring ratios allow to implement different synergies, not different softness that allow various hand and wrist positions.

\section{CONCLUSIONS}

In this work we have presented a modular, soft, underactuated wrist with two DOFs and only one motor. The mechanical design, whose theoretical foundations rely on the framework of soft and adaptive synergies, is modular in order
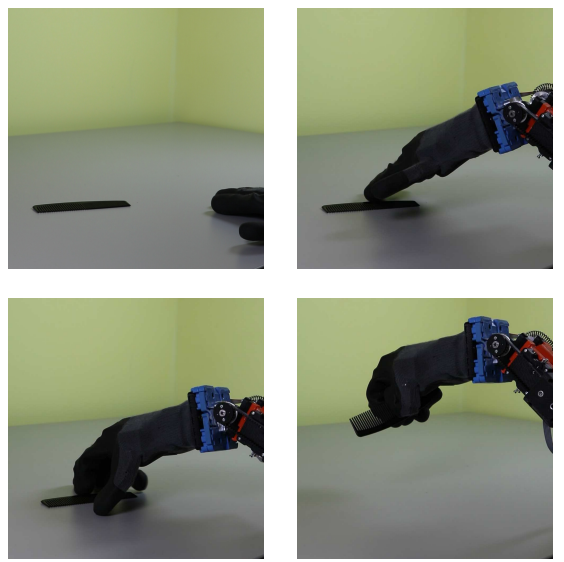

Fig. 12. Example of object grasping with the under-actuated wrist implementing PC1.

to allow the implementation of different under-actuation patterns. In parallel, we also performed experiments with human participants to obtain a preliminary estimation of the most probable pose of the human wrist during the pre-grasp-phase in activities of daily living, i.e. a first estimation of wrist synergies. Finally, we have experimentally demonstrated the capabilities of our prototype and discussed a possible implementation of a preliminarily estimated first human wrist synergy, which was obtained thanks to a suitable choice of the elastic elements. Experiments where our device was used in conjunction with the Pisa/IIT SoftHand to grasp different items show the effectiveness of our solution as a multiplier of manipulation capabilities in humans, also thanks to the softness of the system.

The outcomes of this work opens promising scenarios for prosthetic applications, with the possibility to enhance grasping and manipulation performance in amputees, while keeping the complexity of control as simple as possible and still guaranteeing robustness and movement naturalness the latter representing important novel contributions of soft robotic technologies such as the Pisa/IIT SoftHand and its 
prosthetic evolution.

Furthermore, these results can also be used within the soft manipulation paradigm in industrial applications and humanrobot-interaction, allowing to further multiply the capabilities of soft manipulators.

Future works will aim to investigate more in depth wrist synergies in humans and, consequently, to reduce the dimensions of the wrist prototype - this aspect is especially important in trans-radial amputations for a successful integration of the wrist with the socket and the hand, which could be designed to implement a specific under-actuation pattern devised from human example. Additional experiments will be also performed to further evaluate the effectiveness of the wrist in grasping and manipulation tasks.

\section{REFERENCES}

[1] C. J. van Andel, N. Wolterbeek, C. A. Doorenbosch, D. H. Veeger, and J. Harlaar, "Complete $3 \mathrm{~d}$ kinematics of upper extremity functional tasks," Gait \& posture, vol. 27, no. 1, pp. 120-127, 2008.

[2] N. Yang, M. Zhang, C. Huang, and D. Jin, "Motion quality evaluation of upper limb target-reaching movements," Medical engineering \& physics, vol. 24, no. 2, pp. 115-120, 2002.

[3] S. Ma and A. Feldman, "Two functionally different synergies during arm reaching movements involving the trunk," Journal of neurophysiology, vol. 73, no. 5, pp. 2120-2122, 1995.

[4] N. M. Bajaj, A. J. Spiers, and A. M. Dollar, "State of the art in prosthetic wrists: Commercial and research devices," in 2015 IEEE International Conference on Rehabilitation Robotics (ICORR). IEEE, 2015, pp. 331-338.

[5] C. Gosselin and J. Angeles, "The optimum kinematic design of a spherical three-degree-of-freedom parallel manipulator," Journal of mechanisms, transmissions, and automation in design, vol. 111, no. 2, pp. 202-207, 1989.

[6] F. L. Hammond, R. D. Howe, and R. J. Wood, "Dexterous highprecision robotic wrist for micromanipulation," in Advanced Robotics (ICAR), 2013 16th International Conference on. IEEE, 2013, pp. $1-8$.

[7] M. Bonilla, E. Farnioli, C. Piazza, M. Catalano, G. Grioli, M. Garabini, M. Gabiccini, and A. Bicchi, "Grasping with soft hands," in 2014 IEEE-RAS International Conference on Humanoid Robots. IEEE, 2014, pp. 581-587.

[8] F. Montagnani, M. Controzzi, and C. Cipriani, "Is it finger or wrist dexterity that is missing in current hand prostheses?" IEEE Transactions on Neural Systems and Rehabilitation Engineering, vol. 23, no. 4, pp. 600-609, 2015

[9] — "Preliminary design and development of a two degrees of freedom passive compliant prosthetic wrist with switchable stiffness," in Robotics and Biomimetics (ROBIO), 2013 IEEE International Conference on. IEEE, 2013, pp. 310-315.

[10] H. Takeda, N. Tsujiuchi, T. Koizumi, H. Kan, M. Hirano, and Y. Nakamura, "Development of prosthetic arm with pneumatic prosthetic hand and tendon-driven wrist," in 2009 Annual International Conference of the IEEE Engineering in Medicine and Biology Society. IEEE, 2009, pp. 5048-5051.

[11] P. Louis Goudreau BASc, B. Greg Bush et al., "Two-degree-offreedom powered prosthetic wrist," Journal of rehabilitation research and development, vol. 48, no. 6, p. 609, 2011.

[12] L. Resnik, S. L. Klinger, and K. Etter, "The deka arm: Its features, functionality, and evolution during the veterans affairs study to optimize the deka arm," Prosthetics and orthotics international, vol. 38, no. 6 , pp. 492-504, 2014

[13] "Hormer dorrance friction wrist," http://hosmer.com/, The Hormer Dorrance Corporation.

[14] W. Prout, "Universal wrist system," Mar. 26 1974, uS Patent 3,798,680.

[15] M. G. Catalano, G. Grioli, E. Farnioli, A. Serio, C. Piazza, and A. Bicchi, "Adaptive synergies for the design and control of the pisa/iit softhand," The International Journal of Robotics Research, vol. 33, no. 5, pp. 768-782, 2014

[16] J. Rosen, J. C. Perry, N. Manning, S. Burns, and B. Hannaford, "The human arm kinematics and dynamics during daily activities-toward a
7 dof upper limb powered exoskeleton," in ICAR'05. Proceedings., 12th International Conference on Advanced Robotics, $2005 . \quad$ IEEE, 2005, pp. 532-539.

[17] N. A. Bernstein, "The co-ordination and regulation of movements," 1967

[18] M. Santello, G. Baud-Bovy, and H. Jörntell, "Neural bases of hand synergies," Frontiers in computational neuroscience, vol. 7, p. 23, 2013.

[19] M. Santello, M. Bianchi, M. Gabiccini, E. Ricciardi, G. Salvietti, D. Prattichizzo, M. Ernst, A. Moscatelli, H. Jörntell, A. M. Kappers et al., "Hand synergies: integration of robotics and neuroscience for understanding the control of biological and artificial hands," Physics of life reviews, 2016.

[20] R. J. Bootsma, R. G. Marteniuk, C. L. MacKenzie, and F. T. Zaal, "The speed-accuracy trade-off in manual prehension: effects of movement amplitude, object size and object width on kinematic characteristics,' Experimental brain research, vol. 98, no. 3, pp. 535-541, 1994.

[21] Y. Paulignan, C. MacKenzie, R. Marteniuk, and M. Jeannerod, "The coupling of arm and finger movements during prehension," Experimental Brain Research, vol. 79, no. 2, pp. 431-435, 1990.

[22] C. R. Mason, J. E. Gomez, and T. J. Ebner, "Hand synergies during reach-to-grasp," Journal of Neurophysiology, vol. 86, no. 6, pp. 2896 2910, 2001.

[23] C. Y. Brown and H. H. Asada, "Inter-finger coordination and postural synergies in robot hands via mechanical implementation of principal components analysis," in 2007 IEEE/RSJ International Conference on Intelligent Robots and Systems. IEEE, 2007, pp. 2877-2882.

[24] M. Bianchi, P. Salaris, and A. Bicchi, "Synergy-based hand pose sensing: Optimal glove design," The International Journal of Robotics Research, vol. 32, no. 4, pp. 407-424, 2013.

[25] A. G. Feldman, "Once more on the equilibrium-point hypothesis $(\lambda$ model) for motor control," Journal of motor behavior, vol. 18, no. 1 , pp. $17-54,1986$.

[26] A. Bicchi, M. Gabiccini, and M. Santello, "Modelling natural and artificial hands with synergies," Phil. Trans. R. Soc. B, vol. 366, no. 1581, pp. 3153-3161, 2011.

[27] G. Grioli, M. G. Catalano, E. Silvestro, S. Tono, and A. Bicchi, "Adaptive synergies: an approach to the design of under-actuated robotic hands," in International Conference of Intelligent Robots and Systems - IROS 2012, Vilamoura, Algarve, Portugal, October 7 - 12 2012, pp. $1251-1256$

[28] C. D. Santina, G. Grioli, M. G. Catalano, A. Brando, and A. Bicchi, "Dexterity augmentation on a synergistic hand: the pisa/iit softhand+," in 15th IEEE RAS Humanoids Conference (HUMANOIDS2015), IEEE. Seoul, Korea, November 3 - 5, 2015: IEEE, 2015, pp. 497 - 503.

[29] C. Piazza, C. D. Santina, M. G. Catalano, G. Grioli, M. Garabini, and A. Bicchi, "Softhand pro-d: Matching dynamic content of natural user commands with hand embodiment for enhanced prosthesis control," in IEEE International Conference of Robotics and Automation (ICRA2016), IEEE. Stockholm, Sweden, May 16-21: IEEE, 2016 , pp. $3516-3523$.

[30] M. Benati, S. Gaglio, P. Morasso, V. Tagliasco, and R. Zaccaria, "Anthropomorphic robotics," Biological Cybernetics, vol. 38, no. 3, pp. $125-140,1980$.

[31] E. Biryukova, A. Roby-Brami, A. Frolov, and M. Mokhtari, "Kinematics of human arm reconstructed from spatial tracking system recordings," Journal of biomechanics, vol. 33, no. 8, pp. 985-995, 2000

[32] G. Rab, K. Petuskey, and A. Bagley, "A method for determination of upper extremity kinematics," Gait \&amp; posture, vol. 15, no. 2, pp. 113-119, 2002.

[33] L. Peppoloni, A. Filippeschi, E. Ruffaldi, and C. A. Avizzano, "A novel 7 degrees of freedom model for upper limb kinematic reconstruction based on wearable sensors," in 2013 IEEE 11th international symposium on intelligent systems and informatics (SISY). IEEE, 2013, pp. 105-110.

[34] M. Gabiccini, G. Stillfried, H. Marino, and M. Bianchi, "A data-driven kinematic model of the human hand with soft-tissue artifact compensation mechanism for grasp synergy analysis," in 2013 IEEE/RSJ International Conference on Intelligent Robots and Systems, Nov 2013, pp. 3738-3745.

[35] L. Resnik, L. Adams, M. Borgia, J. Delikat, R. Disla, C. Ebner, and L. S. Walters, "Development and evaluation of the activities measure for upper limb amputees," Archives of physical medicine and rehabilitation, vol. 94, no. 3, pp. 488-494, 2013. 